\title{
Programme de dictée basé sur l'aaprentissage inversé pour remédier à quelques fautes d'orthographe française auprès les étudiants de deuxième année secondaire.
}

\author{
Par \\ Tag Al Din Mohammed Eid \\ Maître de conférences de la méthodologie de FLE à la faculté \\ de pédagogie - Université de Minia. \\ (E.mail :tagemohamed@yahoo.fr)
}

\section{RESUME DE LA RECHERCHE}

Commettre des fautes d'orthographe française est normal et répété soit en classes de FLM soit en classes de FLE . Les enseignants de FLE et aussi les chercheurs dans le domaine de la didactique de FLE ont essayé d'employer plusieurs stratégies afin de remédier à ces fautes de l'orthographe. Comme la stratégie de classe inversée s'impose comme une nouvelle stratégie d'apprentissage de FLE, le chercheur vise à la recherche présente à appliquer programme basé sur la dictée en fonctionnant de stratégie de classe inversée pour remédier aux fautes de l'orthographe française chez les étudiants de deuxième année secondaire. Le chercheur a préparé d'abord le cadre théorique qui traite avec plus de détails les types des fautes d'orthographe, la dicté et aussi la stratégie de classe inversée, ensuite ;il a élaboré les outils de la recherche qui comptent un liste de fautes de l'orthographe, un test de l'orthographe et un programme basée sur la classe inversée. Le chercheur a appliqué le pré test de l'orthographe avant d'apprendre le programme de la recherche aux étudiants de groupe expérimental et après l'apprentissage du programme, il a appliqué le post test de l'orthographe. Le traitement statistique des notes des étudiants ont affirmé l'effet positif de l'emploi de dictée basée sur la classe inversée 
Tag Al Din

Mohammed Eid
Programme de dictée basé sur l'aaprentissage inversé pour remédier à quelques

en faisant la dictée en ligne à la maison et la dictée négociée en classe à remédier aux fautes de l'orthographe française. C'est pourquoi le chercheur recommande de poursuivre la recherche dans le domaine de classe inversée pour améliorer les compétences de la compréhension orale et écrite en FLE.

MOTS CLES : Programme - dictée - stratégie - apprentissage inversé - fautes d'orthographe - français - cycle secondaire

ملخص باللغة العربية

\section{برنامج إملاء قائم على إستراتيجية التصليه المعكوس لتصويب بعض الأخطاء الإملائية فى اللفة الفرنسية للدى طلاب الصف الثانى الثانوى.}

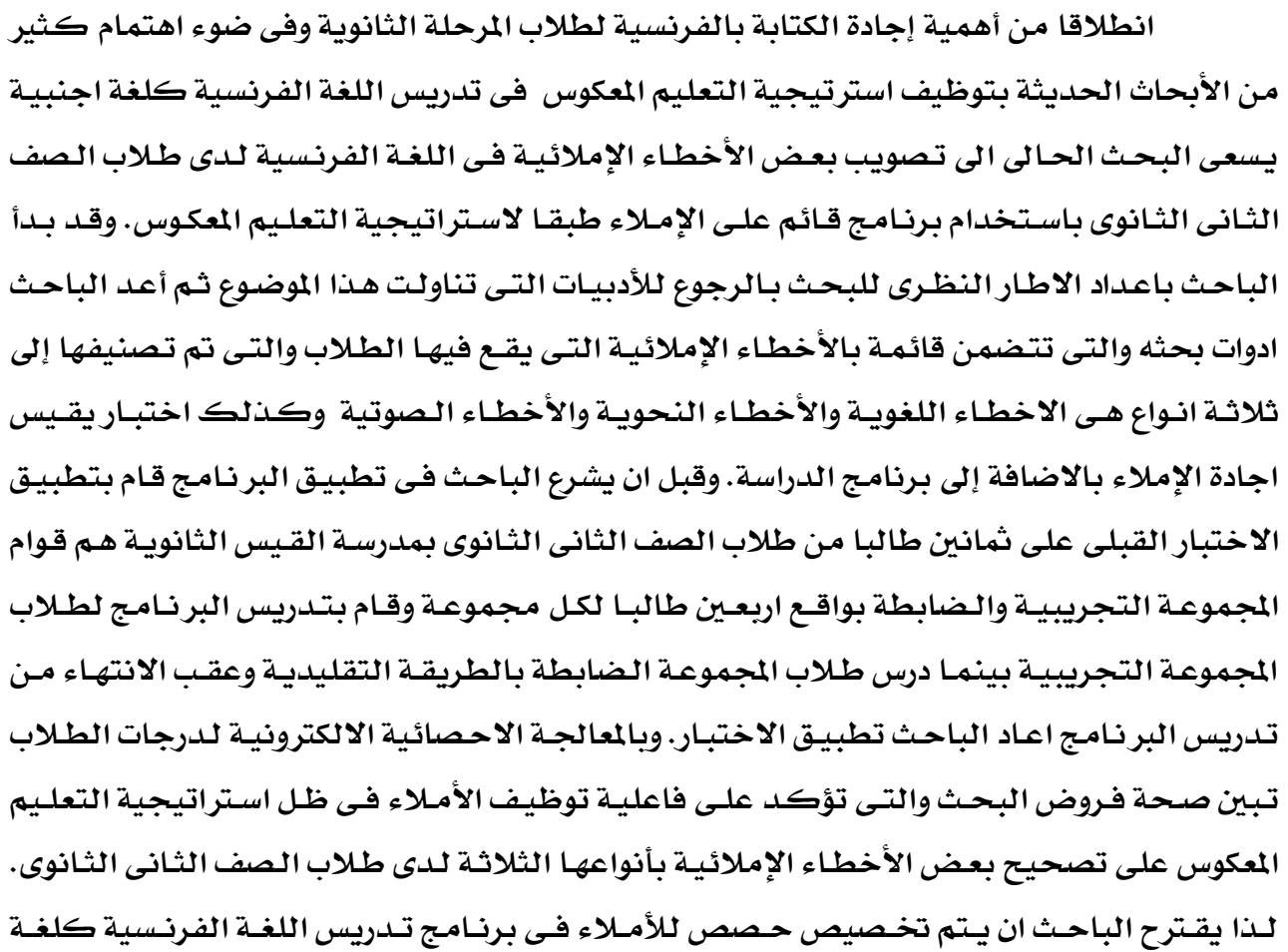




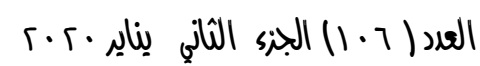



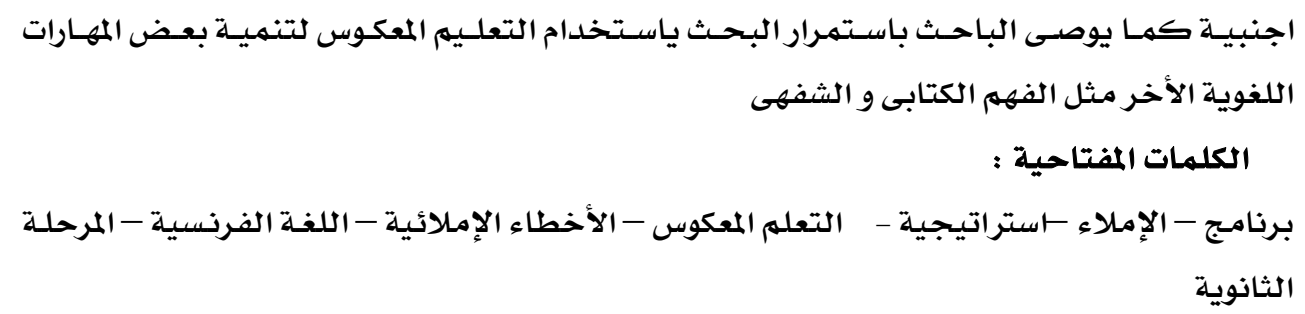

\section{INTRODUCTON}

Ecrire en français sans commettre des erreurs est une compétence qui ne s'acquiert qu'après une longue d'appropriation qui suppose une attention permanente de la part des enseignants. La bonne connaissance de l'orthographe française permet aux apprenants d' écrire correctement, de développer la fluidité de la lecture et aussi d'améliorer la compréhension écrite. L'orthographe française est particulièrement complexe et difficile. soit en ce qui concerne du niveau lexical où le degré d'opacité des correspondances entre les phonèmes et les graphèmes soit en ce qui concerne du niveau grammatical où les accords en genre et en nombre sont sources de nombreuses erreurs.

Comme l'orthographe française n'est pas facile pour les Français, elle est sûrement plus difficile pour un public étranger, surtout si le système orthographique de la langue maternelle des apprenants est très éloigné de celui de la langue française, comme c'est le cas pour la langue arabe.(8). Autrement dit, les difficultés concernant l'orthographe de français comme FLE se diffèrent de celles concernant l'orthographe française comme FLM. Le point de départ de l'apprenant étranger dans son apprentissage de l'orthographe française est ses connaissances dans sa langue 
Tag Al Din

Mohammed Eid
Programme de dictée basé sur l'aaprentissage inversé pour remédier à quelques

maternelle, et surtout, les stratégies d'apprentissage déjà acquises dans cette langue. Pour cette raison, la nature des erreurs orthographiques diffère entre les francophones et les apprenants étrangers.

L'amélioration de l'orthographe exige le développement d'un ensemble de compétences d'ordres différents : compétences phonétiques, phonologiques, grammaticales et syntaxiques. C'est pourquoi, l'acquisition de l'orthographe nécessite un enseignement régulier d'après les stratégies et les activités variées que l'enseignant adapte en classe. La dictée est un exercice, qui est considérée comme moyen permettant le développement et l'acquisition des compétences orthographiques, sous ces différentes formes. Elle représente une pratique idéale pour exercer les apprenants de FLE à améliorer l'orthographe étant donné que cette pratique oblige l'apprenant à spécifier l'identité et la position de chaque lettre de façon précise, alors que les effets lexicaux et contextuels participent à la reconnaissance en lecture. $(8: 12)$

En général, faire des dictées permet un perfectionnement important de la grammaire et de l'orthographe tout en permettant d'enrichir le vocabulaire des apprenants. Car face à un texte lu à haute voix, l'élève doit repérer les pièges en identifiant la nature des mots et choisir la graphie adéquate.(20)

Dans ce contexte, (Isabelle Le Brun \& al 2018) ont constaté l'efficacité de la dictée guidée à développer l'orthographe 


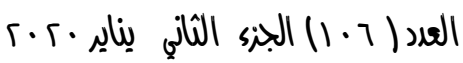



française auprès les élèves âgés de 12 ans par rapport à la dicté classique..(5)

En dépit de son utilité à développer les compétences de l'orthographe, la dictée n'est pas employée en classe de FLE en Egypte. En conséquence, la présente recherche vise à l'employer à remédier aux fautes d'orthographe commises par les apprenants de FLE des écoles secondaire en Egypte en adoptant la stratégie de l'apprentissage inversé.

En effet, la grande majorité des enseignants adaptent le modèle transmissif traditionnel à faire les classes de FLE dans nos institutions éducatives secondaires. Ce modèle exige que l'apprenant doit être attentif, écouter, suivre, imiter, répéter et appliquer.Cette pédagogie traditionnelle transmissive est basée sur deux phases successives : une phase d'acquisition qui se fait en classe et une autre phase d'utilisation des connaissances (l'exercice d'application) qui se déroule, en grande partie hors du temps scolaire.(10)

La stratégie de l'apprentissage inversé renverse ce modèle de l'enseignement traditionnel. En appliquant l'apprentissage inversé ,les étudiants prennent connaissance de la matière à distance en dehors de la classe, principalement au travers de lectures ou de vidéos. Le temps de la classe est alors consacré à un travail plus profond d'assimilation des connaissances au travers de méthodes pédagogiques comme la résolution de problèmes, les discussions ou les débats. 

inversé pour remédier à quelques

Ainsi, l'idée principale de classe inversée est de bousculer les espaces-temps de l'enseignement et de l'apprentissage en proposant les leçons, sous la forme de vidéos, en dehors de l'espace-temps de la classe. Marcel Lebrun (2017) indique que le concept initial de «classe inversée" (Flipped Classroom) a été introduit, il y a une dizaine d'années, par deux enseignants du secondaire en chimie à Colorado aux Etats Unis. (13)

Même si le phénomène des classes inversées est relativement récent, il a été largement discuté en France par les enseignants qui s'y intéressent au cours d'un congrès tenu chaque année depuis 2015 en présence d'un grand nombre de ces enseignants.

Les effets de ces classes inversées sont multiples : elle éveille la motivation des apprenants et aussi des enseignants, elle présent des meilleures appropriations des savoirs formalisés, elle développe quelques compétences disciplinaires et transversales, et elle présente des enseignements et des apprentissages différenciés. L'objectif est de recentrer l'apprentissage autour de l'apprenant en lui permettant les moyens d'être plus autonome. Inverser la classe revient donc à profondément modifier le rôle traditionnel de l'enseignant : ce dernier n'est plus le sachant qui déverse son savoir, mais devient un véritable guide d'apprentissage. Il passe du faceà-face au côte-à-côte, permettant ainsi la mise en place d'une coconstruction des savoirs.(12) 
La stratégie de classe inversée a été expérimenté comme modèle d'apprentissage de FLE. Ziane Khadidja (2017) a employé cette stratégie pour améliorer les compétences de la compréhension et production orale chez les étudiants de licence en algériens.(9)

Le développement accéléré de TICE aux dernières années a bien participé à répandre la pédagogie de classe inversée comme une nouvelle stratégie de l'apprentissage de FLE. Dufour Héloïse indique que deux types d'outils offerts par les nouvelles technologies sont cependant particulièrement pertinents dans un telmodèle: la vidéo et les questionnaires en ligne.(12)

L'emploi des TICE d'une part donne aux apprenants de FLE l'occasion d'après plusieurs sites d'internet de recevoir en ligne à la maison de grandes quantités des connaissances dans toutes les branches de la langue :vocabulaire, grammaire, dialogues, culture etc. D'autre part ,elle aide l'enseignant à faire en classe des activités appliquées qui fortifient la compréhension et développement les compétences des apprenants.

La recherche présente correspond alors avec la nouvelle direction du ministère de l'enseignement en Egypte qui vise à développer l'enseignement au cycle secondaire en employant les tablettes. Le chercheur a pour but d'exploiter un certain nombre de sites d'internet qui présente des dictées en ligne à remédier aux fautes de l'orthographe française chez les apprenants de deuxième année secondaire en leur demandant de faire la dictée chez eux d'après l'écoute d'un contenu téléchargé sur ces sites. Quand ils rentrent en classe 
Tag Al Din

Mohammed Eid
Programme de dictée basé sur l'aaprentissage inversé pour remédier à quelques

,'enseignant les guide à faire la dictée négociée en travaillant en groupe afin de corriger les erreurs commises à maison.

\section{PROBLEMATIQUE DE LA RECHERCHE}

L'apprentissage de l'orthographe française représente un réel problème dans l'enseignement d'une langue écrite et d'ailleurs, on ne cesse pas de parler de la faiblesse du niveau orthographique des apprenants de FLE. Les difficultés des apprenants en orthographe française est un constat que le chercheur a fait pendant son travail comme enseignant de FLE dans une école secondaire pour 14 ans avant d'être nommé comme maître de conférences de la méthodologie à la faculté de pédagogie, Il a observé que la plupart des apprenants exprimaient des difficultés tant en orthographe lexicale qu'en orthographe grammaticale.

Pour bien préciser la problématique de la recherche, il a appliqué un test de dictée aux étudiants de deux classe de l'école secondaire d'Alquaisse, l'école où il travaillait jusqu'en 2012. D'après la correction des papiers des étudiants il a constaté que :

1-Tous les étudiants ont commis des fautes d'orthographe.

2-Les fautes d'orthographe commises par les étudiants sont classifiées sous trois types :

-Fautes de lexique : 1215 fautes. 




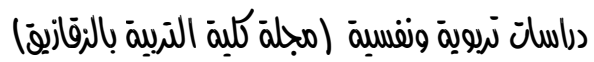

- Fautes de grammaire :827 fautes

-Fautes de phonétique : 1517 fautes.

De plus ,beaucoup de chercheurs ont essayé de préciser les fautes d'orthographe française auprès les apprenants de FLE.

Ruba Ahmed s'intéresse à analyser les fautes orthographiques grammaticales et lexicales en langue française chez des étudiants syriens à l'aide de trois types d'épreuves : la dictée, la rédaction d'un texte narratif et la prise de notes de cours, et ce, en s'appuyant sur un échantillon de 24 étudiants syriens.(8)

Abd El baki Noureddine considère qu'il est pertinent et impératif de déterminer les causes puis proposer une remédiation pour une maitrise des compétences orthographiques par des apprenants qui veulent apprendre le français langue étrangère.(1).

La problématique de cette recherche réside dans une faiblesse au niveau de la dictée auprès les étudiants de la deuxième secondaire ce qui demande de répondre à la question principale suivante :

-Quel est l'effet de l'emploi de la classe inversée à faire une dictée et à corriger les fautes d'orthographe en français ?

De cette question principale, on peut formuler les questions secondaires suivantes : 
Tag Al Din

Mohammed Eid
Programme de dictée basé sur l'aaprentissage inversé pour remédier à quelques

1-Quelles sont fautes d'orthographe principales commises par les étudiants des écoles secondaire ?

2-Comment désigner une liste des fautes d'orthographe principales destinée aux étudiants des écoles secondaire ?

3-Comment élaborer un programme basé sur la dictée en fonctionnant la classe inversée?

4-Quel est l'effet du programme de la recherche à remédier aux fautes d'orthographe commises par les étudiants des écoles secondaire?

\section{LIMITES DE LA RECHERCHE}

1-Cette recherche a été expérimentée aux apprenants de deuxième année secondaire de l'Alquaisse.

2-Le chercheur a analysé les erreurs commises par les apprenants de deuxième année secondaire d'après un test de dictée.

3-Le chercheur a élaboré deux outils : une liste des erreurs d'orthographe et un test d'orthographe.

4-Le programme de la recherche est basé sur l'emploi de classe inversée en faisant la dictée en ligne à la maison et la dicté négociée en classe. 


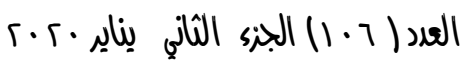

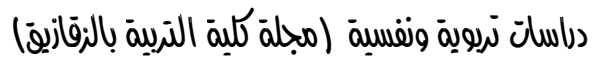

Terminologie de la recherche

Orthographe

Larousse définit l'orthographe comme ensemble de règles et d'usages définis comme norme pour écrire les mots d'une langue donnée. (On distingue l'orthographe d'accord, fondée sur les règles de la grammaire, et l'orthographe d'usage, qui n'obéit pas à des règles précises.) (15)

Selon Nina Catach ,l'orthographe est un système d'écriture et elle le définit comme suit :« La manière d'écrire les sons ou les mots d'une langue en conformité d'une part avec le système de transcription graphique adopté à une époque donnée, d'autre part suivant certains rapport établis avec les autres sous-systèmes de langue (morphologie, syntaxe, lexique,...).(2)

La présente recherche définit l'orthographe comme manière d'écrire les sons et les mots de français d'après un programme basé sur la dictée en ligne à la maison et la correction des fautes en classe.

Dictée

Larousse définit la dictée comme exercice scolaire ayant

pour but l'enseignement et le contrôle de l'orthographe .(17)

Selon Wikipédia,la dictée est l'opération par laquelle une personne lit à haute voix un texte cohérent selon un rythme qui permet à des auditeurs de le copier par écrit. Cette discipline essentiellement scolaire détermine le niveau d'orthographe et de grammaire des élèves.(18)

Dictée négociée 
Tag Al Din

Mohammed Eid
Programme de dictée basé sur l'aaprentissage inversé pour remédier à quelques

La dictée négociée selon Micheline Cellier est définit comme : «Un dispositif pédagogique spécifique centré sur la négociation et la justification, conçue pour laisser une large place à la parole de l'élève »(11)

La présente recherche définit la dictée comme opération dans laquelle, les apprenants copient par écrit un texte cohérent qu'ils écoutent en ligne selon un rythme lent.

Classe inversée

Selon Marcel Lebrun et Julie Lecoq « une flipped classroom , ou classe inversée, est une stratégie pédagogique où la partie transmissive de l'enseignement (exposé, consignes, protocole, etc.) se fait à distance en préalable à une séance en présence, notamment à l'aide des technologies (vidéo en ligne du cours ,lecture de documents papier, préparation d'exercice, etc.) et où l'apprentissage fondé les activités et les interactions se fait en présence (échange entre l'enseignant et les apprenants et entre pairs, projet de groupe, activités de laboratoire, séminaire, débat,...(13 )

La classe inversée est un modèle original qui permet, à partir de concepts pédagogiques anciens, de faciliter l'apprentissage des élèves. C'est également une manière pertinente d'utiliser les nouvelles technologies au service de la pédagogie.(12)

La présente recherche définit la classe inversée comme un modèle d'apprentissage qui permet aux apprenants de faire la dictée à la maison d'après l'écoute d'un document en ligne et de négocier leurs erreurs en groupe en classe. 
PERTINENCE DE LA RECHERCHE.

1- Télécharger la liste de fautes d'orthographe de français commises par les apprenants aux écoles secondaires aux réseaux sociaux pour être à disposition des enseignants et des apprenants en Egypte.

2- Relever l'importance de la dictée comme une activité de remédier aux fautes d'orthographe française en classe de FLE en Egypte

3-Mettre à la disposition des enseignants de FLE une stratégie efficace basée sur la dictée à remédier aux fautes d'orthographe française.

4-Attirer l'attention des responsables du ministère de l'enseignement à l'importance de l'emploi de classe inversée comme une nouvelle stratégie de l'apprentissage de FLE.

\section{HYPOTHESES DE LA RECHERCHE}

1- Il y a une différence significative entre la moyenne des notes des étudiants du groupe expérimental et celle du groupe temoin au test de l'orthographe avant et après l'application du programme en faveur du post test

2- Il y a une différence significative entre la moyenne des notes des étudiants du groupe expérimental au test de l'orthographe avant et après l'application du programme de la recherche en faveur du post test.

\section{METHODOLOGIE DE LA RECHERCHE}

$C$ 'est une recherche quasi expérimentale qui vise à tester l'effet d'une variable indépendante (l'apprentissage inversé) sur une variables dépendante (le niveau de dictée)

\section{ECHANTILLON DE LA RECHERCHE}

Le chercheur a choisi l'échantillon de la recherche parmi les 
Tag Al Din

Mohammed Eid
Programme de dictée basé sur l'aaprentissage inversé pour remédier à quelques

étudiants de l'école secondaire d'Alquaisse. Cet échantillon compte 80 étudiants(garçons - filles) reprtis en deux groupes : un groupe expériemental(40 étudiants) et un groupe témoin (40 étudiants)

1-Le chercheur a choisi le public de la recherche de cette école car ,elle est très proche à l'adresse du chercheur.

2-Il choisi les étudiants de deuxième année parce qu'ils ont acquis un contenu lexical et grmmatical convenable d'après l'apprentissage de FLE en première année.

\section{PROCEDURES DE LA RECHERCHE}

Le chercheur a suivi un certain nombre d'étapes pour achever cette recherche, on peut les résumer comme suit :

1-Formuler le cadre théorique de la recherche en suivant ces étapes :

a) Consulter les études déjà faites dans le domaine de la recherche.

b) Consulter les périodiques et les sites qui touchent au sujet de la

recherche.

c) Traiter les informations concernant les variables de la recherche tels que : l'orthographe française,la dictée et la classe inversée.

d) Relier les thèmes traités d'une manière qui sert le sujet de la recherche.

2- Elaborer une liste des fautes de l'orthographe de FLE commises par les étudiants de deuxième année secondaire:

a) En consultant les références qui s'intéressent à analyser ces erreurs.

b) En appliquant un test de dictée aux étudiants de deuxième année secondaire. 
c) En examinant les fautes commises par les étudiants en faisant la dictée.

3- Elaborer un test d'orthographe française destiné aux étudiants de deuxième année secondaire et msurer statistiqument la validité et la stabilité de ce test.

4- Elaborer un programme basé sur l'emploi de la dictée d'après la classe inversée en suivant ces étapes:

a) Préciser les objectifs du programme.

b) Choisir un certain nombre des passages de dictée téléchargés en ligne sur le site : français facile et podcast fraçais facile.

c) Adapter les textes de dictée choisis aux sujets de livre Club@dos plus 2 que les apprenants de deuxième année secondaire etudient en Egypte.

d) Désigner les activité consacrées de chaque dictée.

e) Déterminer les supports et les stratégie de l'apprentissage du programme.

f) Elaborer le livret d'étudiant et le guide de l'enseignant.

5- Choisir l'échantillon de la recherche qui compte 80 étudiants (garçons et filles) divisés en deux groupes : un groupe expérimental et un groupe témoin.

6-Appliquer le pré test de l'orthographe française aux étudiants de groupe expérimental et témoin.

7) Enseigner le programme aux étudiants de groupe expérimental tandis que les étudiants du groupe témoin ont étudié le même contenu par la méthode traditionnelle.

8) Appliquer le post test de l'orthographe française aux étudiants de groupe expérimental et témoin.

9-Traiter statistiquement les notes des étudiants.

10-Analyser les résultats.

11-Formuler les recommandations et les suggestions de la recherche. 


\section{Le CADRE THEORIQUE DE LA RECHERCHE}

1-L'orthographe française

L'orthographe est un concept impliquant la renaissance d'une norme écrite par rapport à laquelle on juge l'adéquation des formes que réalisent les sujets écrivant une langue. L'orthographe suppose que l'on distingue des formes correctes et des formes incorrectes dans une langue écrite contrairement à la graphie qui implique par référence une norme grammaticale ».(16)

La majorité des problèmes d'apprentissage du FLE se situent au niveau de l'orthographe parce que la plupart des apprenants ne sont pas capables d'écrire n'importe quel texte sans faire des erreurs et la plupart entre eux ne savent pas comment les corriger. Cela nous conduis à présenter les éléments de l'orthographe française selon le point de vue de Coach.

\section{1-1. Eléments de l'orthographe française}

D'après Catach, le système orthographique français contient trois éléments inséparables : les phonogrammes (qui ont une dimension phonologique), les morphogrammes et les logogrammes (qui ont tous deux une dimension idéographique) on développe avec plus de détails les trois éléments (2)

\section{1-1.1.Les phonogrammes}

C'est la transcription des sons où les unités de l'écrit, appelées les graphèmes, notent les unités de l'oral appelées 




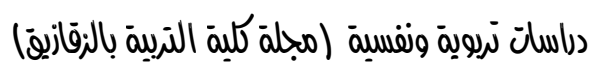

les phonèmes (Catach, 1980).

Quatre types de phonogrammes existent :

1-1.1.1 Lettre simple : quand il $y$ a une seule lettre qui correspond à un phonème $:(b=/ b /)$.

1-1.1.1.2 Lettre simple à signe auxiliaire : une seule lettre (mais qui a un accent ou un tréma ou une cédille) qui correspond à un phonème : fête, ça, maïs, etc.

1-1.1.3 Digrammes : groupe de deux lettres qui correspondent à un seul phonème :ai (mais), ei (neige) pour $/ \square /$, ph pour /f/, ou pour /u/, etc.

1-1.1.4Trigrammes : groupe de trois lettres qui correspondent à un seul phonème :eau pour /o/, ain pour / /, etc. (Riegel et al., 1994).

Fayol indique qu'il y a dans un système alphabétique, les lettres de l'alphabet sont associées à des phonèmes. Et il ajoute que la correspondance entre les unités sonores et les unités orthographiques n'est cependant pas systématique : le phonème / $k$ /, par exemple, peut s'orthographier « $c$ ", «qu ", «k ", "ck» ou encore «ch » (Fayol, 2003). En fait, la complexité du système orthographique français renvoie à plusieurs phénomènes : il existe plus de phonèmes que de lettres : certains graphèmes $(\mathrm{ch} ; \mathrm{s}$; $\mathrm{c}$; etc.) correspondent à plusieurs phonèmes ; certains phonèmes peuvent se transcrire de plusieurs manières. Cette complexité entraine des difficultés pour les étrangers, mais aussi pour les Français. (Fayol, 2006). 
Tag Al Din

Mohammed Eid
Programme de dictée basé sur l'aaprentissage inversé pour remédier à quelques

1-1.2 Les morphogrammes

Au niveau grammatical, les graphèmes sont porteurs des informations d'ordre lexical ou grammatical. Ils indiquent les marques de genre, de nombre et de flexion verbale (comme la terminaison), ainsi que les marques lexicales des familles de mots dérivés (Catach, 1980).

\section{1-1.2.1 Les morphogrammes grammaticaux :}

Ce sont des désinences supplémentaires qui s'ajoutent aux mots variables pour apporter des indications de genre et de nombre (classes nominales), de personne et de nombre (verbes) » (Riegel et al., $1994:$ 125).

\section{1-1.2 .2 Les morphogrammes lexicaux :}

Ce sont des marques internes ou finales fixes, intégrées au mot, qui servent essentiellement à relier graphiquement un radical à ses dérivés (tard, tarder, tardif, retarder, attarder) ou bien le masculin au féminin (petit/petite ; grand/grande). Ils maintiennent l'unité des familles des mots et constituent des marques graphiques de sens ». (Riegel et al., 1994 : 126).

1-1.2 .3-Les logogrammes ou « figure des mots »

Les graphèmes permettent de différencier les mots homophones grammaticaux (ce/ ces/ ses) ou lexicaux (compte/ conte/ comte). (Catach, 1980b). Ces distinctions reposent sur des lettres muettes qui sont contestées quand 


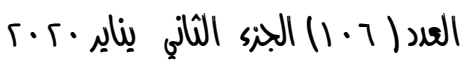

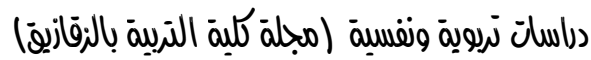

leur fonction distinctive ne semble pas nécessaire. Elles aident le lecteur en permettant d'identifier visuellement le sens mais elles causent pourtant une gêne à celui qui écrit (Riegel et al., 1994).

\subsection{Difficultés du système orthographique français}

Fayol (2001) regroupe les problèmes en orthographe en trois ensembles qui correspondent tous au système français :

1.2.1-La mise en correspondance entre phonèmes et graphèmes. Dans un système alphabétique, transcrire un mot nécessite le recours au procédé de la lecture à travers l'analyse de la forme sonore du mot en phonèmes puis la mise en correspondance de ces phonèmes avec des graphèmes.

1.2.2-Le système non-transparent, en français, l'apprentissage des correspondances phonèmes/graphèmes ne suffit pas pour lire et écrire pratiquement tous les mots car les correspondances entre sons et lettres peuvent être irrégulières (les doubles consonnes ne sont pas phonologiquement marquées, par exemple). On dit de ce système qu'il est non-transparent.

1.2.3-La morphologie sémantique : les marques morphologiques en français, surtout du nombre mais aussi du genre, sont souvent inaudibles, comme le « $s$ » et le « $x$ » des noms et des adjectifs ainsi que le «nt $»$ à la fin des verbes. Pour la marque du genre, les mots sont généralement marqués auditivement, alors que pour le nombre, la plupart des mots ont une marque écrite mais pas de marque orale. 
Tag Al Din

Mohammed Eid
Programme de dictée basé sur l'aaprentissage inversé pour remédier à quelques

Ce sont des marques silencieuses qui sont acquises au moment où les élèves apprennent à écrire. Ces marques ne sont donc prises en compte que quand l'apprentissage de la lecture et de l'écriture commence (Fayol, 2001).

1-3 Typologie des fautes d'orthographe

Selon Nina Catach, les erreurs forment six catégories :

\subsection{1-Les fautes à dominante phonétique}

Elles sont dues à une mauvaise production orale. Par exemple : l'apprenant qui écrit maman, parce qu'il ne sait pas que l'on prononce [mamà].

Et pour remédier ce type d'erreur, il est nécessaire d'assurer l'orale.

\subsection{2-Les fautes à dominante phonogrammique}

Ce genre d'erreurs correspond à un oral correct, un écrit erroné, les phonogrammes sont les graphèmes qui sont chargés de transcrire les différents phonèmes. Par exemple : [à] correspond à les phonogrammes : en, em, an, am. C'est comme l'apprenant qui transpose l'oral en écrit par le biais d'archi graphèmes

\subsection{3-Les fautes à dominante morphogrammique}




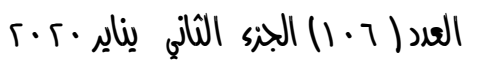

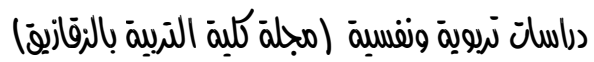

Les morphogrammes (on graphème non chargés de transcrire des phonèmes) sont des suppléments graphiques qui assurent diverses fonctions :

Marques finales de liaison : exemple : la finale muette d'un mot Marques grammaticales, comme :

Les morphogrammes de genre : e,

Les morphogrammes de nombre : $\mathbf{s}, \mathbf{x}$.

Les morphogrammes verbaux : e, s, e. Marques finales de dérivation : grand-grandeur. Marques internes de dérivation : main, manuel.

1.3.4-Les fautes à dominante morphogrammique sont donc tantôt lexicales, tantôt grammaticales (dans ce cas, elles portent sur les accords et ne relèvent pas d'une logique immuable).

1.3.5-Les fautes concernant les homophones (ou encore logogrammes)

Ceux-ci peuvent être lexicaux (chant/champ), ou grammaticaux (c'est/s'est) ils peuvent aussi relever du discours.

\subsection{6-Les fautes concernant les idéogrammes}

Considéré comme idéogramme, tout signe qui ne relève pas uniquement de l'alphabet, c'est le cas des majuscules, des signes de ponctuation. 
Tag Al Din

Mohammed Eid
Programme de dictée basé sur l'aaprentissage inversé pour remédier à quelques

Les erreurs concernant les lettres non justifiables d'un enseignant

Lettres étymologiques, exemple : sculteur/sculpteur, rume/rhume.

\section{2- La dictée}

La dictée est un exercice, qui peut être exploité comme un moyen efficace au développement et à l'acquisition des compétences orthographiques, sous ces différentes formes.

\subsection{Les formes de dictée}

Abd El bakinoureddine El-kantara(2017) classifie la dictée sous plusieurs formes (1)

\subsubsection{La dictée traditionnelle :}

La dictée traditionnelle est un exercice au cours duquel les apprenants doivent produire, sans aucune aide extérieure, les formes graphiques d'un texte choisi et à haute voix par l'enseignant, suivie parfois d'une correction magistrale, elle est ainsi conçue, est contestée par l'ensemble des didacticiens de l'orthographe, mais beaucoup plus un instrument d'évaluation.

\subsubsection{La dicté sans faute :}

Elle est un type d' exercice au cours duquel l'enseignant choisit un texte à orthographier aux apprenants comme la 


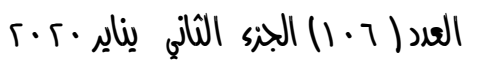



dictée traditionnelle. Mais les objectifs visés sont radicalement différents, de même que les modalités de mise en ouvre par l'enseignant, contrairement à la dictée traditionnelle, la dictée sans faute ne remplit aucune fonction d'évaluation sommative, elle vise à renforcer leur compétences, elle aide les apprenants dans les productions orthographiques.

\subsubsection{La dictée aidée :}

Lorsque l'enseignant relit la dictée, les apprenants ont le droit et l'obligation de se servir des outils : dictionnaire, grammaire, cahier de dictée, leçons, et même de travailler par deux apprenants (binôme).

\subsubsection{L'autodictée :}

Cette dictée base sur la mémorisation, c'est-à-dire pour travailler la mémoire en classe et mettre en rapport avec d'autre discipline (leçon à apprendre par cœur).

\subsubsection{La dictée à choix multiple :}

Avec cette dictée en donnant des propositions différentes selon les apprenants, on peut changer et différencier l'activité, en plus, cette dictée est intéressante car la forme juste d'un mot est parfois "stockée " en mémoire, mais n'arrive pas à être récupérée, entre trois propositions, l'apprenant pourra retrouver le mot recherché.

2.1.6.La dictée à trous : 
Tag Al Din

Mohammed Eid
Programme de dictée basé sur l'aaprentissage inversé pour remédier à quelques

De même avec la dictée à trous, on peut cibler l'évaluation sur des points précis, comme la gestion des accords en nombre au sein du groupe nominal.

\subsubsection{La dictée en ligne :}

C'est un type de dictée basée sur l'emploi de TICE, elle peut être faite à distance à la maison. La recherche présente adapte ce type de dictée dont l'idée principale exige que l' enseignant choisit les textes de dictée téléchargés sur les sites d'internet et demande aux apprenants de faire la dictée à la maison. Ils ont le droit de réécouter le texte original plusieurs fois, utiliser le dictionnaire, ou les livres de grammaire.

\subsubsection{La dictée négociée :}

La dictée négociée selon Micheline Cellier est définit comme : «Un dispositif pédagogique spécifique centré sur la négociation et la justification, conçue pour laisser une large place à la parole de l'élève ".C'est à dire, cette dictée provoque des échanges entre les apprenants et qui les obliges à faire des choix, et les justifier.(11)

Ève Langevin a réalisé et testé la dictée négociée en utilisant la négociation de la forme et l'interaction entre les pairs. Cette dictée est issue des stratégies d'enseignementapprentissage métacognitives. Elle a pour objet de faire émerger, de développer et de mettre en situation les 


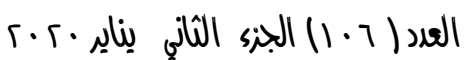

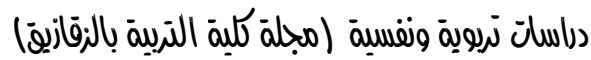

connaissances ainsi que la conscience métalinguistique et la conscience morphologique des élèves relativement à l'orthographe lexicale.(3)

Joëlle Thébault, indique que la dictée négociée peut être exécutée en France aux classe de CM1 et CM2 à visée purement évaluative et elle permet aux élèves d'apprendre à se relire en se posant les bonnes questions.(14)

Ainsi la recherche présente vise à désigner des activités de dictée en ligne sous la forme de choix multiple, dictée à trous, dictée à erreurs et dictée pure que les apprenants font à la maison. Mais en classe, ils faisons la dictée négociée ,c'est pourquoi on présente plus de détails qui lui concernent.

\subsection{La technique de dictée négociée.}

Après la dictée d'un texte et la relecture individuelle en ligne à la maison, l'enseignant divise la classe en petits groupes de deux à quatre élèves. Chaque groupe doit rendre une seule copie au professeur, rédigée à partir de la confrontation des dictées individuelles de ses membres. L'objectif est d'amener les élèves à argumenter, justifier et expliciter oralement leurs choix orthographiques individuels pour aboutir à un consensus. La copie remise à l'enseignant est le fruit de cet accord, la version "négociée " de la dictée. La correction est collective : le processus de confrontation est réitéré, mais cette fois en classe entière. L'occasion de rediscuter et d'expliquer les notions qui posent problème. Cette tâche peut être exécutés sur plusieurs étapes : 
Tag Al Din

Mohammed Eid
Programme de dictée basé sur l'aaprentissage inversé pour remédier à quelques

2.2.1. Recherche ( individuelle )

Après avoir écrit un texte sous la dictée en lige, chaque apprenant a un temps pour se relire, réfléchir, se corriger à l'aide des outils dont il dispose.

\subsubsection{Négociation ( groupes de 3 ou 4 )}

Par groupes, les apprenants confrontent leurs productions et se mettent d'accord pour réécrire le texte sur une affiche ou une nouvelle feuille. Ils sont amenés à justifier leurs choix, argumenter en ayant recours aux outils ou à leurs connaissances, expliciter leurs stratégies.

\subsubsection{Synthèse ( classe entière )}

Il s'agit de proposer un choix après comparaison des productions affichées. Ce travail se fait avec tout le groupe classe. L'échange et la réflexion sont menés sous la tutelle du maître. Un apprenant donne son avis sur un élément de la $1^{\text {ère }}$ phrase. Ceux qui ne sont pas d'accord peuvent faire une autre proposition et la défendre. On écrit au tableau ce qui est proposé. On laisse pour la dernière phase les problèmes d'orthographe qui restent sans solution.

On procède ainsi pour les autres éléments de la $1^{\text {ère }}$ phrase que les élèves interrogés veulent mettre en débat puis idem pour la $2^{\text {ème }}$ phrase, etc.

\subsubsection{Institutionnalisation}

L'enseignant valide la production finale en soulignant les fautes et en faisant référence à la règle ou à la phrase modèle. 


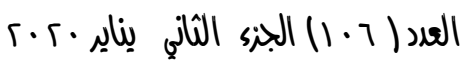



3. La stratégie de l'apprentissage inversé

L'apprentissage inversé est un moyen de rendre les apprenants plus acteurs et producteurs de leur savoir. Ils visionnent les leçons à leur propre rythme. La progression du travail dépend de leur activité : ce sont les élèves qui permettent réellement l'inversion de la classe. La bonne marche de cette dernière dépend en effet de leur volonté d'être actifs en cours.

\subsection{Les avantages de l'apprentissage inversé .}

Héloïse présente un certain nombre d'avantages de la classe inversée

3.1.1-Elle permet de gagner temps en classe pour passer plus de temps sur les activités : le temps de classe qui était réservé au cours magistral ou dialogué est maintenant consacré à l'accompagnement des élèves.

3.1.2-Elle représente une bonne occasion de tutorat par les pairs : La classe inversée bénéficie bien des avantages du travail en groupe : les étudiants apprennent les uns des autres, ce qui bénéficie aux élèves quelque soit leur niveau. Les apprenants faibles bénéficient du soutien et des explications de leurs pairs, tandis que les apprenants de niveau avancé, en expliquant à leurs pairs, approfondissent leur compréhension et renforcent leur apprentissage.

3.1.3-Elle fait du temps de classe plus agréable : d'une part les enseignants, externaliser la partie magistrale du cours cela permet d'éviter le côté lassant et répétitif de l'enseignement. Les interactions avec les apprenants permettent un cours beaucoup plus vivant et personnalisé. D'autre part, les élèves étant 
Tag Al Din

Mohammed Eid
Programme de dictée basé sur l'aaprentissage inversé pour remédier à quelques

en activité permanente, si le volume sonore est plus élevé du fait du travail en groupes, ils sont beaucoup moins susceptibles de perturber le déroulement de la classe. Enfin, le travail en groupe représente un environnement moins stressant pour les apprenants.

4-Elle développe l' autonomie des apprenants : La classe inversée fait les apprenants être responsables de leur apprentissage. en consultant des ressources pour ensuite fournir un travail en classe.(12)

\section{2- Règles de l'apprentissage inversé}

Voici quelques conseils adressés à ceux qui souhaitent employer la l'apprentissage inversé :

3.2.1 Se familiariser avec le concept de la classe inversée

Cela paraît évident, mais il est important de se faire sa propre opinion avant de se lancer. Il n'existe pas un modèle unique de classe inversée : c'est un concept que chaque enseignant met en pratique de manière différente.

\subsubsection{Inverser petit-à-petit}

Il n'est pas nécessaire de tout inverser dès la première année (ni au final d'ailleurs). Cela représente énormément de travail, surtout à faire d'un seul coup. Choisissez une section et lancez-vous. Au fur et à mesure, vous accumulerez le matériel nécessaire pour inverser de plus en plus de chapitres et de classes. 
3.2.3 Expliquer aux élèves comment travailler en classe inversée :

Au final, ce sont réellement les élèves qui inversent la classe : s'ils viennent en classe non préparés, ils ne peuvent pas participer aux activités d'application de manière efficace. La classe inversée représente un changement de paradigme pour eux aussi qu'il est utile de leur expliquer. Selon l'âge et l'enjeu, la proportion d'élèves qui résistent très fortement à la classe inversée peut varier. De manière générale, plus les élèves sont âgés et habitués au système traditionnel, plus ils ont l'impression qu'en classe inversée, le professeur n'enseigne pas. De la même manière, si l'année s'achève sur un examen, ou pour les élèves qui sont très attachés à avoir d'excellentes notes, l'anxiété de ne pas être dans un modèle classique peut être plus forte.

\subsubsection{Travailler en collaboration}

Inverser sa classe avec un collègue a de nombreux avantages : en plus d'avoir un partenaire avec lequel échanger des idées, cela réduit la charge de travail inhérente au projet et permet de produire des vidéos de meilleure qualité.

\subsubsection{Intégrer des évaluations à toutes les étapes}

Sans doute l'évaluation améliore l'apprentissage.

Pratiquement pour la classe inversée, il est très productif de coupler les vidéos avec des questionnaires très simples. Ces questionnaires ont plusieurs avantages : en plus d'améliorer l'apprentissage, les points qui leur sont 
Tag Al Din

Mohammed Eid
Programme de dictée basé sur l'aaprentissage inversé pour remédier à quelques

attribués motivent les élèves à faire le travail

préparatoire.(19)

\subsection{Employer la classe inversée à faire la dictée}

La technique de classe inversée suivie dans la recherche présente.

La recherche présente vise à employer la classe inversée en exploitant la dictée pour remédier aux erreurs d'orthographe française en suivant les étapes. 1-L'enseignant choisit un certain nombre de textes de dictée téléchargés sur les sites d'internet.

2-Il demande aux étudiants de faire la dictée à la maison en écoutant les textes en ligne.

3-Quand, les étudiants rentrent en classe, l'enseignant les guide à exercer la dictée négociée en groupes.

4- Il corrige les copies de chaque groupe et leur distribuent les copies corrigées.

5-Il montre au tableau les difficultés que les apprenants n'arrivent pas à corriger.

\section{CADRE PRATIQUE}

1-Elaboration des outils de la recherche

Le chercheur a élaboré trois outils afin de vérifier les hypothèses de la recherche, on présente la technique de préparer ces outils avec plus de détails.

1-1 Premier outil : Liste des fautes de l'orthographe 
Cette liste est destinée aux étudiants de deuxième année secondaire.

Pour élaborer cette liste, le chercheur a suivi ces étapes :

Il a consulté les références et les sites d'internet qui s'intéresse à analyser les erreurs de l'orthographe français.

2-Il a appliqué un test de dictée à 80 étudiants de deuxième année de l'école secondaire d'Alquaisse (à Minia). Ce test comptait deux textes de dictée (annexe 1)

3-L'enseignant a corrigé le test de dictée en soulignant les fautes.

4-Il classifie les fautes sous les types déjà traités dans le cadre théorique.

1.1.1Classification types de fautes d'orthographe

Les fautes d'orthographe française commises par les apprenants peuvent être classifiées sous trois types principaux :

A-Fautes de lexique

-Fautes d'épellation

-Fautes d'employer les accents

-Confondre les types d'accents

-Ne pas mettre le cédille ou le trima.

- Le (à) et le (a)

-Les mots à double consonne.

-Les terminaisons des mots

B-Fautes de phonétique

-Les fautes de phonétique commises par les apprenants concernent.

- Les voyelles et semi voyelles

-La liaison

-L'élision 
Tag Al Din

Mohammed Eid
Programme de dictée basé sur l'aaprentissage inversé pour remédier à quelques

-Les homonymes.

-les lettres nasales.

C-Fautes de grammaire

Ces fautes de grammaire concernent

-La conjugaison des verbes ;

-La terminaison des verbes conjugués et l'infinitif :er, ez, ai, ais, ait, aient,é ;ée,és,ées

-L'accord de genre et de nombre ;

-L'accord du participe passé ;

-la distinction du masculin et de féminin en ce qui concerne les articles définis, les adjectifs démonstratifs et les pronoms personnels d'objet direct.

\subsubsection{Importance de la liste de l'orthographe}

Pour faire cette liste plus utile et plus pertinente le chercheur peut :

1-Publier cette liste sur les réseaux sociaux afin de faire les enseignants de FLE d'en profiter.

2- Mettre cette liste à la disposition des futurs enseignants qui étudient à la faculté de pédagogie.

3-Orienter les responsables du ministère de télécharger cette liste sur le site de la Banque des connaissance pour que les enseignants et les apprenants des écoles secondaire puissent la consulter.

2-Deuxième outil : Le test d'orthographe française / 80 pts

Ce test est destiné aux étudiants de deuxième année secondaire, le chercheur a élaboré ce test (Annexe 3) afin de - 400 - 
mesurer l'effet de la dictée en fonctionnant la stratégie de classe inversée sur la remédiation des erreurs de l'orthographe française.

On a élaboré ce test à l'aide d'un certain nombre de sites électroniques consacrés à l'apprentissage de français comme français facile, et podcast français facile.

\section{2-1.La construction du test}

\section{Ce test compte huit questions :}
2.1.1-
La
première
question:

(10 pts)

Cette question vise à mesurer la remédiation des fautes qui concernent l'emploi les mots d'interrogation qui exigent la distinction de genre et de nombre (Quel (s)(le)(les)
2.1.2
La
deuxième
question

(10 pts)

Cette question mesure la correction des fautes d'employer les terminaison des verbes conjugués ,des adjectifs et des participes passé.
2.1.3
La
troisième
question (10 pts)

Le but de cette question est de mesurer la capacité de l'emploi des homonymes et d'éviter les fautes commises par les étudiants en écoutant celles-ci.
2.1.4
La
quatrième
question

(10 pts)

Cette question a pour but de mesurer la compétence de l'emploi l'accord de genre et de nombre des adjectifs.
2.1.5
La
quatrième
question

(10 pts) 
Tag Al Din

Mohammed Eid
Programme de dictée basé sur l'aaprentissage inversé pour remédier à quelques

La cinquième question du test vise à mesurer la compétence de l'emploi l'accord du participe passé.

1.6 La $\quad$ sixième $\quad$ question (10 pts)

Cette question présente un texte qui compte dix fautes d'orthographe, l'étudiant de sa part doit découvrir ces erreurs et les citer dans le tableau au dessous du texte afin de les éviter.

$\begin{array}{llll}1.7 & \text { La } & \text { septième } & \text { question }\end{array}$ (10 pt)

C'est un exercice de dictée qui vise à remédier à quelques fautes d'orthographe concernant la le lexique, la phonétique et grammaire.
1.8
La
huitième
question

(10 pts)

C'est un exercice de rédaction qui demande aux étudiants de traiter un sujet. En écrivant l'article, les étudiants doivent tenir en compte les compétences de l'orthographe.

\subsection{Les critères de correction}

Le chercheur a mis un modèle de correction pour corriger le test.

En ce qui concerne aux exercices de dictée et de rédaction, il a mis

un certain nombre de critères pour les corriger

\subsection{Durée du test}


Pour calculer la durée du test, le chercheur a appliqué le test à vingt étudiants, puis, il a calculé le temps que tous les étudiants ont duré et il a divisé la durée totale sur le nombre des étudiants.

Durée du test $=$ La durée totale de tous les étudiants $=$

20

$1260=63$ minutes $=$ presque une heure 20

\section{2-4.La stabilité du test}

Pour constater la stabilité du test, le chercheur a appliqué le test à vingt étudiants de deuxième année de l'école secondaire d'Alquaisse puis, ils utilisé le programme statistique SPSS à calculer la valeur d'Alpha de Cronbach .79 qui est indiquée dans le tableau №1 et cette valeur montre que le test est stable.

\section{Tableau №1}

Statistiques de fiabilité du test de l'orthographe française.

\begin{tabular}{|ll|c|c|}
\hline & $\mathrm{N}$ & $\%$ \\
\hline Observ & Valide & $\mathbf{2 0}$ & $\mathbf{1 0 0 . 0}$ \\
ations & Exclue $^{\mathrm{a}}$ & $\mathbf{0}$ & $\mathbf{. 0}$ \\
& Total & $\mathbf{2 0}$ & $\mathbf{1 0 0 . 0}$ \\
\hline
\end{tabular}


Tag Al Din

Mohammed Eid
Programme de dictée basé sur l'aaprentissage inversé pour remédier à quelques

Statistiques de fiabilité

\begin{tabular}{|c|c|}
\hline Alpha de Cronbach & Nombre d'éléments \\
\hline .79 & 2 \\
\hline
\end{tabular}

\section{2-5.La validité du test}

Le chercheur a calculé la valeur de la validité du test d'après la valeur d' d'Alpha de Cronbach indiquée dans le tableau :

La validité du tesst $=$ d'Alpha de Cronbach

$$
.79=.89
$$

Cette valeur de validité affirme que le test est valable.

3- Elaboration du programme de la recherche.

Ce programme compte dix leçons basées sur l'emploi de la dictée selon la stratégie de classe inversée à remédier les fautes d'orthographe. Le chercheur a désigné ce programme en suivant quelques étapes :

1- Consulter un certain nombre des sites électroniques français qui présentent des textes variés de dictée sous plusieurs formes soit dictée à choix multiple, dictée à trous, dictée qui compte des fautes et dictée pure. D'après ces sites : français facile, le point de FLE, podcast français facile et ictee.orthodidacte.com.

2- Choisir un certain nombre de textes de dictée dont le contenu convient avec le contenu le livre Club@dos plus 2 que les apprenants de deuxième année secondaire étudient en Egypte. 


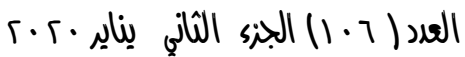



3- Formuler des activités linguistiques sur le contenu de dictée, ces activités exercent les étudiants à écrire correctement sans commettre des fautes d'orthographe.

4- Mettre les objectifs généraux du programme.

5- Mettre les objectifs spéciaux à chaque leçon.

6- Elaborer le livret de l'étudiant(Annexe 4) qui compte :

a- La présentation des types des fautes d'orthographe commises par les étudiants de deuxième année secondaire d'après le test de dictée déjà appliquée à ceux-ci.

b-L'importance de la dictée à remédier les fautes d'orthographe.

c- Les sites d'internet auxquels les étudiants doivent naviguer pour avoir le contenu des activités de la dictée.

d- La technique de déroulement le travail soit à distance à la maison soit en classe.

e- Dix leçons de dictée.

6-Elaborer le guide de l'enseignant (Annexe 5) qui compte :

a- Les objectifs généraux du programme.

b- La stratégie de travail

c- Les supports

d- Les objectifs spéciaux de chaque leçon.

e- Les textes originaux de dictée.

f- La correction des exercices.

\section{3-1 La construction du programme}

Ce programme compte dix leçons qui traitent des textes de types variés de dictée : dictée à choix multiple, dictée à trous, dictée contenant des erreurs et dictée pur. Le chercheur a 
Tag Al Din

Mohammed Eid
Programme de dictée basé sur l'aaprentissage inversé pour remédier à quelques

désigné les leçons du programme selon la graduation du niveau du contenu présenté du simple su complexe.

Les leçons sont organisées comme suit :

3.1.1.Leçon (1) : Dictée à choix multiple

Titre : Au jardin

Objectifs général : Distinguer les homonymes.

Site : https://www.francaisfacile.com/exercices/exercice-francais-2/exercice-

francais-66962.php

3.1.2. Leçon (2) : Dictée à trous

Contenu lexical : La vie à la campagne et la vie en ville

Site https://www.podcastfrancaisfacile.com/podcast/avantages-

ville-campagne.html

Objectif général

Compléter une dictée en ligne

\subsubsection{Leçon (3) : Dictée à trous}

Contenu lexical : Vocabulaire de la boutique

Site https://www.podcastfrancaisfacile.com/podcast/dictee-vocabulaire-

boutique.html

.Objectif général : Compléter une dictée en ligne

3.1.4. Leçon (4) : Dictée à trous

Contenu lexical : La nuit des musées 




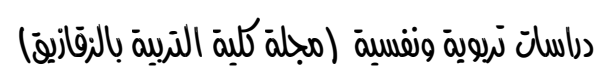

Site https://www.podcastfrancaisfacile.com/podcast/la-nuitdes-musees.html

Objectif général : Compléter une dictée en ligne

\subsubsection{Leçon (5) : Dictée à erreurs}

Contenu lexical : Lycéen

Site :https://www.francaisfacile.com/exercices/exercice -francais-2/exercice-francais-15643.php

Objectif général : Corriger les erreurs d' une dictée en ligne

3.1.6. Leçon (6) : Dictée à erreurs

Contenu lexical : Un professeur sympathique

Site : https://www.francaisfacile.com/exercices/exercice-francais-2/exercicefrancais-68372.php

Objectif général : Corriger les erreurs d' une dictée en ligne

3.1.7. Leçon (7) : Dictée à erreurs

Contenu lexical : Les deux amies

Site : https://www.francaisfacile.com/exercices/exercice-francais2/exercice-francais-120975.php

Objectif général : Corriger les erreurs d' une dictée en ligne

3.1.8. Leçon (8) : Dictée ouvert

Contenu lexical : Les sons - é, è, e, ai

Site :

https://dictee.orthodidacte.com/dictee/dictee-fle-5-sons-e-accent-aigu-e-accentgrave-e-a/contenu/dictee-fle-5-sons-e-accent-aigu-e-accent-grave-e-ai-phrasesguillaume-terrien 
Tag Al Din

Mohammed Eid
Programme de dictée basé sur l'aaprentissage inversé pour remédier à quelques

Objectif général : Rédiger des phrases de dictée en les écoutant en ligne.

3.1.9. Leçon (9) : Dictée ouvert Contenu lexical : Les sons - é, è

Site :

https://dictee.orthodidacte.com/dictee/dictee-fle-4-sons-e-accent-aigu-e-accentgrave-phr/contenu/dictee-fle-4-sons-e-accent-aigu-e-accent-grave-phrasesguillaume-terrien

Objectif général : Rédiger des phrases de dictée en les écoutant en ligne.

\subsubsection{Leçon (10) : Dictée ouvert}

Contenu lexical : Les voyelles nasales an, in, on

Site :

https://dictee.orthodidacte.com/dictee/dictee-fle-2-sons-voyelles-nasales-an-inon-phrase/contenu/dictee-fle-2-sons-voyelles-nasales-an-in-on-phrasesguillaume-terrien

Objectif général : Rédiger des phrases de dictée en les écoutant en ligne.

\section{EXPÉRIMENTATION DE LA RECHERCHE}

Cette recherche a été expérimentée aux étudiants de deuxième année secondaire à l'école secondaire d'Alqaisse au deuxième semestre en 2018. L'expérimentation a duré douze séquences : une séquence par semaine.

Le chercheur a suivi les étapes suivantes: 
1- Le chercheur a consacré la première séquence pour a-Reconnaître les étudiants en expliquant l'idée de sa recherche qui est basée d'une part sur le travail individuel à la maison en employant n'import quel moyen de communication connecté à l'internet : ordinateur, tablette, Ipad, ou un portable pour faire la dictée en ligne. D'autre part, sur le travail en classe en faisant la dictée négociée qui vise l'autocorrection développant la justification.

b-Demander aux étudiants de coopérer avec lui et d'être ponctuels.

c-Relever l'importance de l'orthographe.

d-Présenter les types des fautes d'orthographe communes à la production écrite des étudiants.

e-Appliquer le pré/test de l'orthographe.

2-Le chercheur a appliqué les dix leçons du programme de la recherche en dix séquences, chaque leçon s'est déroulée en deux séances :

La première séance:

Avant de travailler individuellement à la maison, l'enseignant distribue aux étudiants en classe une feuille de papier contenant le site électronique qui compte le texte de dictée et les activités linguistiques désignées sur ce texte.

Les étudiants de leur part, ils naviguent sur le site d'internet et écoutent le texte de dictée d'un rythme lent ,ensuite, ils font les activités et enfin, ils écoutent la dictée pour vérifier leurs réponses. 
Tag Al Din

Mohammed Eid
Programme de dictée basé sur l'aaprentissage inversé pour remédier à quelques

La deuxième séance:

Cette séance est basée sur la dictée négociée qui se fait en classe comme suit :

a-Au début de la séance le chercheur divise les étudiants en groupes.

b- Chaque groupe corrige les erreurs de dictée sous une forme de discussion ou de négociation justifiée.

c- De la même façon, ils corrigent les activités qui suivent la dictée.

d-L'enseignant accueille la version finale de la dictée de chaque groupe et la correction des activités en remerciant tous les groupes pour leur travail.

e-Il corrige le travail de tous les groupes.

f- Il présente au tableau les fautes que tous les groupes n'arrivent pas à les corriger.

g-Il rédige le texte original de la dictée au tableau et la corrections des exercices.

4-Les étudiants du groupe témoin apprennent par la méthode traditionnelle que l'enseignant adapte en classe.

5-Dans la douzième séance, le chercheur a appliqué le post/test de l'orthographe.

\section{Les problèmes de l'expérimentation.}

1-Certains étudiants se plaignent de pas avoir d'outils de communication à la maison et certains autres ont un outil de communication mais pas connecté à l'internet. Pour résoudre à ce problème, le chercheur leur a conseillé d'aller 


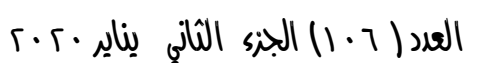



à la salle d'informatique qui se trouve à l'école ou à n'importe quel lieu.

2-Certains étudiants trouvent de difficultés lexicales et grammaticales pendant le travail à la maison. Le chercheur a conseillé à ces étudiants d'avoir référence aux livres ou sites d'internet qui présentent du contenu lexical ou grammatical.

\section{ANALYSE DES RESULTATS}

L'application de la recherche présente vise à vérifier les deux hypothèses de la recherche.

Pour la première hypothèse :

1- Il y a une différence significative entre la moyenne des notes des étudiants du groupe expérimental et celle du groupe témoin au test de l'orthographe avant et après l'application du programme en faveur du post test.

Le chercheur a pu vérifier cette hypothèse par deux manières :

Premièrement, il a analysé les fautes commises par les étudiants du groupe expérimental et celles commises par les étudiants de groupes témoin qui sont indiquées au tableau№ 3.

En observant les nombres des fautes d'orthographe indiqués dans ce tableau, nous pouvons montrer que :

- Pour les fautes de lexique, le nombre de ces fautes commises par les étudiants du groupe expérimental s'est changé de 427 à 94 tandis que le nombre de mêmes fautes 
commises par les étudiants du groupe témoin s'est changé de 439 à 319.

- A l'égard du nombre des fautes de la phonétique faites par les étudiants du groupe expérimental, il s'est changé de 516 à 113 alors que le nombre des fautes commises par leurs collègue de groupe témoin s'est un peu changé de 521 à 371 .

- En ce qui concerne des fautes de grammaire commises par les étudiants du groupe expérimental a diminué de 346 à 74 tandis que le nombre de ces fautes commises par les étudiants du groupes témoin a diminué de 383 à 293

D'après les nombres de types de fautes, nous pouvons constater qu'il une amélioration observable aux capacités de l'orthographe des étudiants du groupe expérimental par rapport à celles du groupe témoin.

Deuxièmement:Le chercheur a traité statistiquement les notes des étudiants du groupe expérimental et celles des étudiants du groupe témoin au poste test de l'orthographe par le programme SPSS et il a obtenu les résultats indiqués dans le tableau № 2.

Tableau № 2 : Résultats du groupe expérimentalet témon au post test de l'orthographe

\begin{tabular}{|c|c|c|c|c|c|c|c|}
\hline $\begin{array}{c}\text { Test du } \\
\text { vocabulaire }\end{array}$ & $\mathbf{N}$ & Ма & $\mathbf{E}$ & DL & C & $\mathbf{T}$ & $\mathbf{S}$ \\
\hline $\begin{array}{c}\text { Groupe } \\
\text { expérimental }\end{array}$ & \multirow{2}{*}{40} & 61.60 & 11.957 & \multirow{2}{*}{59} & \multirow{2}{*}{.} & \multirow{2}{*}{8.698} & \multirow{2}{*}{$\begin{array}{c}\text { au } \\
\text { niveau } \\
\text { de } 0 . \\
01\end{array}$} \\
\hline $\begin{array}{l}\text { Groupe } \\
\text { témoin }\end{array}$ & & 39.63 & 7.732 & & & & \\
\hline
\end{tabular}


$\mathrm{N}=$ nombre des étudiants.

Ma=moyenne arithmétique.

$E=$ écart type.

$D L=$ degré de liberté.

$\mathrm{C}=$ Corrélation

$T=$ valeur de (t) test.

$\mathrm{S}=$ significative

Ces résultats nous montre que la valeur de moyenne arithmétique des notes étudiants du groupe expérimental est 61.60 et la valeur de celle-ci des étudiants du groupe témoin est 39.63.Si on a comparé aussi l'écart type des notes des étudiants de deux groupes, on peut constater qu'il y a une augmentation à la valeur de l'écart type des notes des étudiants du groupe expérimental (11.96) par rapport à celle-ci des étudiants du groupe témoin(7.73) Selon les résultats indiqués dans le tableau, on peut affirmer que la valeur de $T$ (8.70) est significative au niveau de.01. Cela indique que les activités de la dictée que les étudiants faites à la maison selon stratégie de classe inversée employée et aussi la dictée négociée suivie en classe ont participé à remédier aux fautes de l'orthographe française par rapport à la méthode traditionnelle que l'enseignant de FLE suit en classe de deuxième année secondaire en apprenant le livre de $c l u b$ @dos plus2.

Pour le deuxième hypothèse

2- Il y a une différence significative entre la moyenne des 
notes des étudiants du groupe expérimental au test de l'orthographe avant et après l'application du programme en faveur du post test.

Pour vérifier cette hypothèse,le chercheur a suivi deux étapes :

Première étape :

Il a analysé les réponses des étudiants au test de l'orthographe, le chercheur a classifié les fautes commises par les étudiants et a fait le bilan des fautes qui est indiqué au tableau №3. En observant ce tableau, Nous pouvons constater que le nombre de fautes du lexique commises par les étudiants du groupe expérimental avant l'application de programme est 427. Ce nombre a diminué après l'application à 94.

Pour les fautes de phonétique, les étudiants du groupe expérimental ont fait 516 fautes avant l'apprentissage du programme alors qu'ils ont fait $\mathbf{1 1 3}$ fautes seulement après l'apprentissage du programme.

En ce qui concernent les fautes de grammaire, les étudiants ont commis 364 fautes avant l'expérimentation tandis qu'ils n'ont commis que 76 après l'expérimentation. Tous ces indices affirment que l'apprentissage du programme basé sur la dictée en fonction de la stratégie de classe inversée a un effet très positif à remédier aux types des fautes d'orthographe française. 
Tableau №3

Fautes commises par les étudiants

\begin{tabular}{||c|c|c|c|c|}
\hline \multirow{2}{*}{$\begin{array}{c}\text { Types de } \\
\text { fautes }\end{array}$} & \multicolumn{2}{|c|}{$\begin{array}{c}\text { Nombres des fautes } \\
\text { du groupe } \\
\text { expérimental }\end{array}$} & \multicolumn{2}{|c|}{$\begin{array}{c}\text { Nombres des fautes du } \\
\text { groupe témoin }\end{array}$} \\
\cline { 2 - 5 } & Pré test & Post test & Pré test & Post test \\
\hline $\begin{array}{c}\text { Fautes } \\
\text { de lexique }\end{array}$ & 427 & 94 & 439 & 319 \\
\hline $\begin{array}{c}\text { Fautes } \\
\text { de phonétique }\end{array}$ & 516 & 113 & 521 & 371 \\
\hline $\begin{array}{c}\text { Fautes } \\
\text { de grammaire }\end{array}$ & 364 & 76 & 383 & 293 \\
\hline Total & 1307 & 283 & 1343 & 983 \\
\hline
\end{tabular}

Deuxième étape :

Le chercheur a employé le programme électronique de statistique SPSS à traiter les notes des étudiants du groupe expérimental au pré test et au post test de l'orthographe. Il a obtenu les résultats indiqués au tableau№ 4

En observant ceux-ci, nous pouvons constater que la valeur de la moyenne arithmétique des notes des étudiants du groupe expérimental avant l'application de la recherche est 33.93 alors que cette valeur est devenue 61.60. De plus ce tableau nous montre que la valeur de l'écart type des notes des étudiants du groupe expérimental avant l'application est presque 9.27,tandis que cette valeur est devenue presque 11.96 après l'application. Nous pouvons constater enfin que la valeur de $T$ est presque 13.9. cette valeur est significative au niveau de .01 
Tag Al Din

Mohammed Eid
Programme de dictée basé sur l'aaprentissage inversé pour remédier à quelques

Tous les résultats indiqués dans le tableau nous conduit à la validité de la première hypothèse et par conséquent l'efficacité de la stratégie employée à remédier aux fautes de l'orthographe française.

Tableau № 4

Résultats du groupe expérimental avant et après

l'expérimentation

\begin{tabular}{|c|c|c|c|c|c|c|c|}
\hline $\begin{array}{c}\text { Test du } \\
\text { vocabulaire }\end{array}$ & $\mathbf{N}$ & Мa & $\mathbf{E}$ & DL & C & $T$ & $\mathbf{S}$ \\
\hline Post test & \multirow[b]{2}{*}{40} & 61.60 & 11.957 & \multirow[b]{2}{*}{59} & \multirow[b]{2}{*}{.317} & \multirow[b]{2}{*}{13.895} & \multirow{2}{*}{$\begin{array}{c}\text { au } \\
\text { niveau } \\
\text { de } 0 . \\
01\end{array}$} \\
\hline Pré test & & 33.93 & 9.266 & & & & \\
\hline
\end{tabular}

$\mathrm{N}=$ nombre des étudiants.

Ma=moyenne arithmétique.

$E=$ écart type.

$D L=$ degré de liberté.

$\mathbf{C}=$ Corrélation

$T=$ valeur de (t) test.

$\mathrm{S}=$ significative . 


\section{RECOMMANDATIONS ET SUGGESTIONS}

A la lumière des résultats de la recherche présente qui a affirmé l'effet de la stratégie de l'apprentissage inversé à remédier aux fautes de l'orthographe française, le chercheur recommande aux futurs chercheurs de poursuivre la recherche basée sur l'emploi de cette stratégie pour développer :

- l'apprentissage de quelques règles grammaticales françaises.

-l'acquisition du vocabulaire ;

-La compréhension de la lecture en français..

Le chercheur suggère que les enseignants de FLE aux écoles secondaires en Egypte adaptent la stratégie de classe inversée basée sur l'emploi des TICE que le Ministère de l'Enseignement vise à généraliser.

Le chercheur suggère aussi que les enseignants de FLE aux écoles et les professeurs aux départements de FLE aux facultés de langues, emploient la dictée de français dans les salles des cours.

\section{Bibliographic}

\section{Livres et Thèses}

1-Abd El bakinoureddine El-kantara: La dictée négociée facteur de la remédiation des erreurs orthographiques dans les productions écrites en FLE,Cas des apprenants de la 3 ème A.M, Mémoire du diplôme de master académique,Universite Mohamed Khider - Biskra faculte des lettres et des langues, 2017,p :8. 
Tag Al Din

Mohammed Eid
Programme de dictée basé sur l'aaprentissage inversé pour remédier à quelques

2-Catach Nina, L'orthographe Française, traité théorique et pratique, Nathan, 1980, p.16.

3-Ève Langevin : Les effets de la dictée négociée sur le gain en orthographe lexicale d'étudiants adultes allophones, mémoire de maitrise de linguistique, Université du Québec à Montréal,2008,p.

4-Fayol, M. (2006). Apprendre l'orthographe. Le cas du français. Repéré le 2 janvier 2013 à http://www.signesed.com/interventionM Fayol.pdf

5-Isabelle Le Brun, et al : Améliorer l'orthographe chez les élèves de 10 à 13 ans : entrainement par dictées guides,érudit Diffusion numérique : 4 mai 2018,https://www.erudit.org/fr/revues/ncre/2016-v19-n3ncre03625/1045177ar/ conulté le 28sept 2018.

6-Marcel Lebrun - Julie Lecoq : classes inversées, enseigner et apprendre à l'endroit, Réseau Canopé .2015.p16.

7-Riegel, M., Pellat, J.-C. \& Rioul, R. (1994). Grammaire méthodique du français. paris : Puf.

8-Ruba Al Ahmad: Les erreurs orthographiques des étudiants syriens en FLE. Analyse et propositions pédagogiques, Université Stendhal - Grenoble 3, Mémoire de master 2 recherche, 2014

9-Ziane Khadidja :Développer autrement la compétences de la production orale par le biais de la classe inversée,Cas des -418 - 
étudiants de $3^{\text {ème }}$ année de licence de français, mémoire du master académique ,Université de Mohamed Boudiaf,M'sila,2017.

10-Brame, C., (2013). Flipping the classroom. Vanderbilt University Center for Teaching. Consulté le 15 juillet 2016 sur http://cft.vanderbilt.edu/guides-sub-pages/flipping-theclassroom/conulté le 20 sept 2018

11-Cellier Micheline, Réflexion sur les différents types de dictées, disponible sur : http://www.ac.nice.fr/iencannet/ien/ortho/difftypesdedicteesp df consulté le: 26/10/2018.

12-Héloïse Duffour (2014) :La classe inversée, Technologie N 193,septembre-octobre 2014 P.45

13-Marcel Lebrun :Les classes inversées, un phénomène précurseur pour «l'école " à l'ère numérique, Université Catholique de Louvain, Belgique, Revue internationale Méthodal, $N^{0} 1$ - Mai 2017.

14-Joëlle Thébault : La dictée négociée :une bonne idée ? pbliée le 28 janvier 2015 sur le site : https://www.vousnousils.fr/2015/01/28/dictee-negociee-unebonne-idee-561661 consultée 28 Mars 2019.

Dictionnaires 
Tag Al Din

Mohammed Eid
Programme de dictée basé sur l'aaprentissage inversé pour remédier à quelques

15-Dictionnaire Larousse: $\underline{\mathrm{h} t \mathrm{tps}: / /}$

www.larousse.fr/dictionnaires/ francais/ orthographe/56605

16-Dubois Jean, Dictionnaire de linguistique, Larousse, Paris, Janvier 2001, p.337.

Sitographie

17-

https://whttps://fr.wikipedia.org/wiki/Dict\%C3\%A9e

18ww.larousse.fr/dictionnaires/francais/dict\%C3\%A

9e/

25353

19-http://www.laclasseinversee.com/

20-https://www.superprof.fr/blog/apprendre-lefrancais-en-classe/ 